\title{
ESTUDOS SOBRE ALIMENTAÇÃO DE ESCOLARES PANORAMA BRASILEIRO
}

\author{
SCHOOL FEEDING STUDIES: BRAZILIAN PANORAMA
}

\author{
Jamille Marinho Brazil, Ana Cristina Santos Duarte
}

Universidade Estadual do Sudoeste da Bahia - UESB

\begin{abstract}
This study aimed to investigate the scientific production on food and school in Brazil. For this, a descriptive and bibliometric research conducted in Brazilian Digital Library of Theses and Dissertations was developed. Initially they found 69 works, however after the analysis of abstracts 49 jobs were defined according to the theme. Of these 49 papers, they were 36 dissertations and 13 theses. It was observed that the issue in question has been developed in graduate programs and by researchers, particularly professional nutrition. Therefore it is important that researchers publish the results coming from their dissertations and theses so that they can contribute to science and society.
\end{abstract}

Key words: Eating Habits; Nutrition Education; School.

\section{Resumo}

Este estudo teve como objetivo investigar a produção científica acerca da alimentação de escolares no Brasil. Para isso, foi desenvolvida uma pesquisa descritiva e bibliométrica, realizada na Biblioteca Digital Brasileira de Teses $e$ Dissertações. Foram encontrados inicialmente 69 trabalhos, porém, após a análise dos resumos, apenas 49 trabalhos estavam de acordo à temática delimitada. Desses 49 trabalhos, foram 36 dissertações e 13 teses. Observou-se que o tema em questão vem sendo desenvolvido nos programas de pós-graduação e pelos pesquisadores, principalmente com os profissionais da nutrição. Logo, é fundamental que os pesquisadores divulguem os resultados oriundos de suas dissertações e teses, para que os mesmos possam contribuir para a ciência e a sociedade.

Palavras-chave: Hábitos Alimentares; Educação Nutricional; Escola. 


\section{Introdução}

A alimentação é um dos fatores primordiais para o crescimento, o desenvolvimento e a manutenção do corpo humano com saúde. Hábitos alimentares saudáveis consistem em uma alimentação equilibrada em quantidade e qualidade de nutrientes, levando-se em consideração a necessidade fisiológica de cada indivíduo, aspectos socioeconômicos, culturais e ambientais ${ }^{1}$.

Os hábitos alimentares são formados durante a infância, os quais são influenciados principalmente pela família e pelo ambiente escolar, sendo estes os primeiros grupos sociais de contato da criança ${ }^{2}$. Hábitos alimentares inadequados desenvolvidos na infância geralmente perpetuam nas próximas fases e estão associados ao aumento de doenças crônicas, como obesidade ${ }^{3}$.

No Brasil, o aumento de sobrepeso e o aumento de obesidade em crianças são notórios. Segundo a Pesquisa de Orçamentos Familiares 2008-2009, cerca de 33,5\% das crianças, entre 5 e 9 anos, estão com excesso de peso e 14,3\% na obesidade $^{4}$. Desse modo, a alimentação inadequada é referenciada como uma das possíveis causas para o aumento de sobrepeso e obesidade, como, por exemplo, o consumo de alimentos ultra processados, ricos em carboidratos refinados, gorduras saturadas e trans $s^{5}$.

Além do elevado consumo de alimentos nutricionalmente pobres, tem se observado em crianças a diminuição no consumo de cereais, tubérculos, raízes e grãos integrais, e baixa ingestão de frutas e legumes. Esses alimentos são ricos em fibras, vitaminas e minerais que contribuem para o desenvolvimento saudável e para a prevenção das doenças crônicas ${ }^{6}$.

Nesse contexto, a educação nutricional durante a infância é fundamental para incorporação de hábitos alimentares saudáveis, como o consumo de frutas, legumes e vegetais, a fim de contribuir para a promoção da saúde ${ }^{7}$. Estudos mostram que atividades de educação nutricional, envolvendo pais, crianças e professores, contribuem significativamente para a melhora na alimentação da criança ${ }^{8}$.

Deste modo, a escola é um lócus privilegiado para abordar temas sobre a alimentação e, assim, contribuir para a formação de hábitos alimentares saudáveis. A educação nutricional é capaz de promover saúde para os indivíduos e prevenir doenças causadas por alimentação inadequada, o que se reflete não apenas no seu estado atual, mas também para o futuro ${ }^{9}$.

Pensando na escola como um ambiente importante para formação de hábitos alimentares saudáveis, o governo tem desenvolvido estratégias para trabalhar com a temática, como o Programa Nacional de Alimentação Escolar (PNAE), que institui um vínculo entre alimentação e escola ${ }^{10}$, e o Programa Saúde na Escola (PSE), o qual traz o conceito de educação em saúde para promoção da qualidade de vida dos escolares ${ }^{11}$.

Porém, na escola se explana muito pouco sobre hábitos alimentares e alimentos de modo geral. Habitualmente, tal conhecimento é trabalhado de forma limitada, já que os professores abordam apenas as fontes de minerais e vitaminas dos alimentos. Faz-se pouca relação entre alimentos, saúde, indivíduos e sociedade, gerando uma lacuna no entendimento entre noções do "saudável instituído" e dos hábitos alimentares ${ }^{12}$.

Nesse contexto, é evidente a importância do levantamento da produção científica sobre alimentação e educação nutricional de crianças escolares, a fim de vislumbrar o cenário brasileiro sobre o tema, observando os avanços e as limitações na educação e na saúde. Desse modo, o presente trabalho objetivou investigar a produção científica em dissertações e teses acerca da alimentação de escolares no Brasil.

\section{Metodologia}

O presente trabalho é uma revisão bibliométrica, do tipo descritivo e de análise documental da produção científica acerca da alimentação de escolares. 0 estudo bibliométrico tem sido utilizado para analisar de forma quantitativa a produção científica, o que permite a percepção do seu panorama, assim como a existência de lacunas para o desenvolvimento de futuros estudos ${ }^{13}$.

Para a busca das produções científicas sobre o tema em estudo, optou-se pela base de dados da Biblioteca Digital Brasileira de Teses e Dissertações (BDTD), visto que a mesma compreende os sistemas de informação de teses e dissertações existentes nas instituições de ensino de pesquisa do Brasil $^{14}$. Os descritores utilizados na busca foram advindos dos Descritores em Ciência da Saúde (DeCS), que são: educação nutricional; alimentação; e escolares. Foi utilizado o operador booleano "AND".

A pesquisa utilizou como critério de inclusão a abrangência temporal entre os anos de 
2010 e 2015. De modo que, só foram incluídas no presente estudo as dissertações e teses publicadas nesse período. As dissertações e teses em duplicata e aquelas que não se enquadravam no tema em estudo foram adotadas como critério de exclusão.

Ademais, foram avaliados os resumos das dissertações e teses elegíveis para o estudo, conforme dados bibliométricos, que foram: o tipo do trabalho, ano, formação profissional do autor principal, instituição de ensino, regiões geográficas brasileiras (sul/sudeste/norte/nordeste/centro-oeste) e produção científica oriunda do trabalho.

É pertinente ressaltar que os dados referentes à formação profissional dos pesquisadores e o meio de divulgação da produção científica foram coletados através de consulta no currículo Lattes dos autores.

Após a leitura dos resumos e análise dos currículos Lattes dos autores, as informações pertinentes foram digitalizadas em planilhas no programa Microsoft Excel, versão 2011. A organização das informações coletadas por meio de planilhas possibilitou a construção do banco de dados e, assim, a análise descritiva do mesmo, como a distribuição de frequência absoluta e relativa.

Não houve a necessidade de aprovação de Comitê de Ética em Pesquisa (CEP), pois se trata de uma pesquisa bibliográfica e os dados foram coletados em plataforma de domínio público. Logo, não ocorreu envolvimento direto de seres humanos como participantes da pesquisa.

\section{Resultados e Discussões}

Foram identificados 69 trabalhos, nos quais foram analisados o título e o resumo. Após a análise, houve a exclusão de 20 trabalhos, sendo 1 duplicado e 19 não relacionados com o tema principal em estudo. Dessa forma, a amostra foi composta por 49 trabalhos, sendo 36 dissertações e 13 teses, os quais abordavam sobre educação nutricional, alimentação e escolares (Figura 1).

Figura 1: Processo esquematizado da revisão bibliométrica.

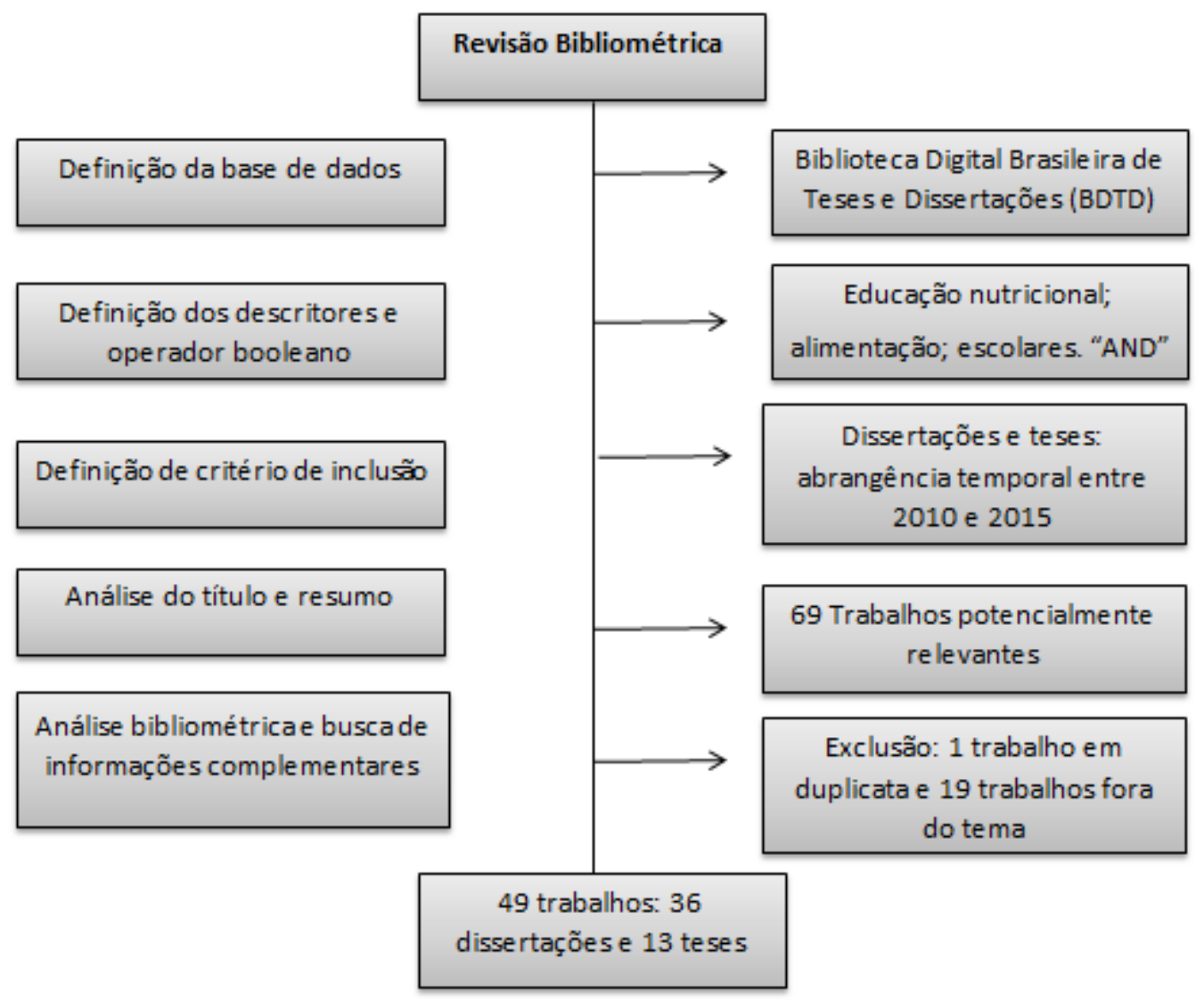

Conforme descreve a Figura 2, a produção de trabalhos acerca da temática foi muito pequena em 2010, sendo contabilizado apenas 1 trabalho. Porém, no ano de 2011 começa a 
crescer (n=10; 20,5\%). Logo em seguida há uma diminuição no número de trabalhos ( $n=5 ; 10,2 \%)$, sendo que em $2013(n=10 ; 20,5 \%)$ volta a crescer e estabiliza até $2015 \quad(n=9 ; \quad 18,4 \%)$.

Figura 2. Distribuição da produção de dissertações e teses segundo o ano de publicação. Em relação à formação profissional dos pesquisadores, a maioria era nutricionista. Entretanto, foi encontrado profissional de outras áreas estudando sobre a temática em questão (Tabela 1).

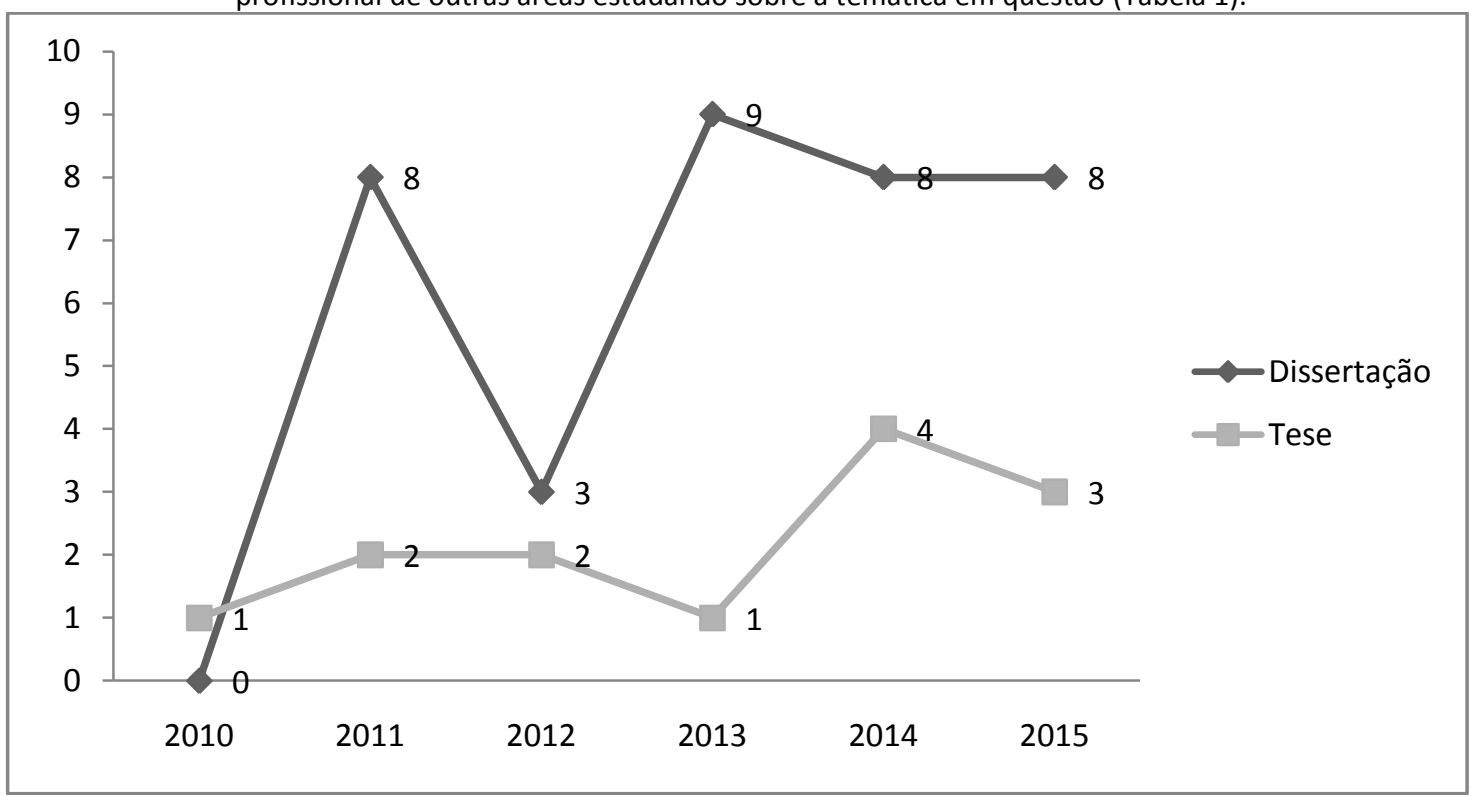

Tabela 1. Formação profissional dos autores dos trabalhos em estudo.

\begin{tabular}{lcc}
\hline Formação profissional & $\mathbf{N}$ & $\%$ \\
\hline Nutrição & 42 & 86 \\
Administração & 01 & 2 \\
Comunicação Social & 01 & 2 \\
Economia Doméstica & 01 & 2 \\
Educação física & 01 & 2 \\
Engenharia Agrícola & 01 & 2 \\
Pedagogia & 01 & 2 \\
História & 01 & 2 \\
\hline Total & & $\mathbf{1 0 0 , 0}$ \\
\hline
\end{tabular}

No que tange às instituições de ensino e às regiões geográficas, observa-se que a região Sul ( $n=18 ; 36,7 \%$ ) lidera o ranking nos trabalhos sobre educação nutricional, alimentação e escolares, sendo que a Universidade Federal do Rio Grande do Sul ( $n=6 ; 12,2 \%$ ) se destacou mais na região e no Brasil com o maior número de trabalhos. É relevante evidenciar que não foi encontrado nenhum trabalho sobre a temática na região Norte, conforme descreve a Tabela 2 
Tabela 2. Distribuição conforme a região geográfica das instituições de ensino.

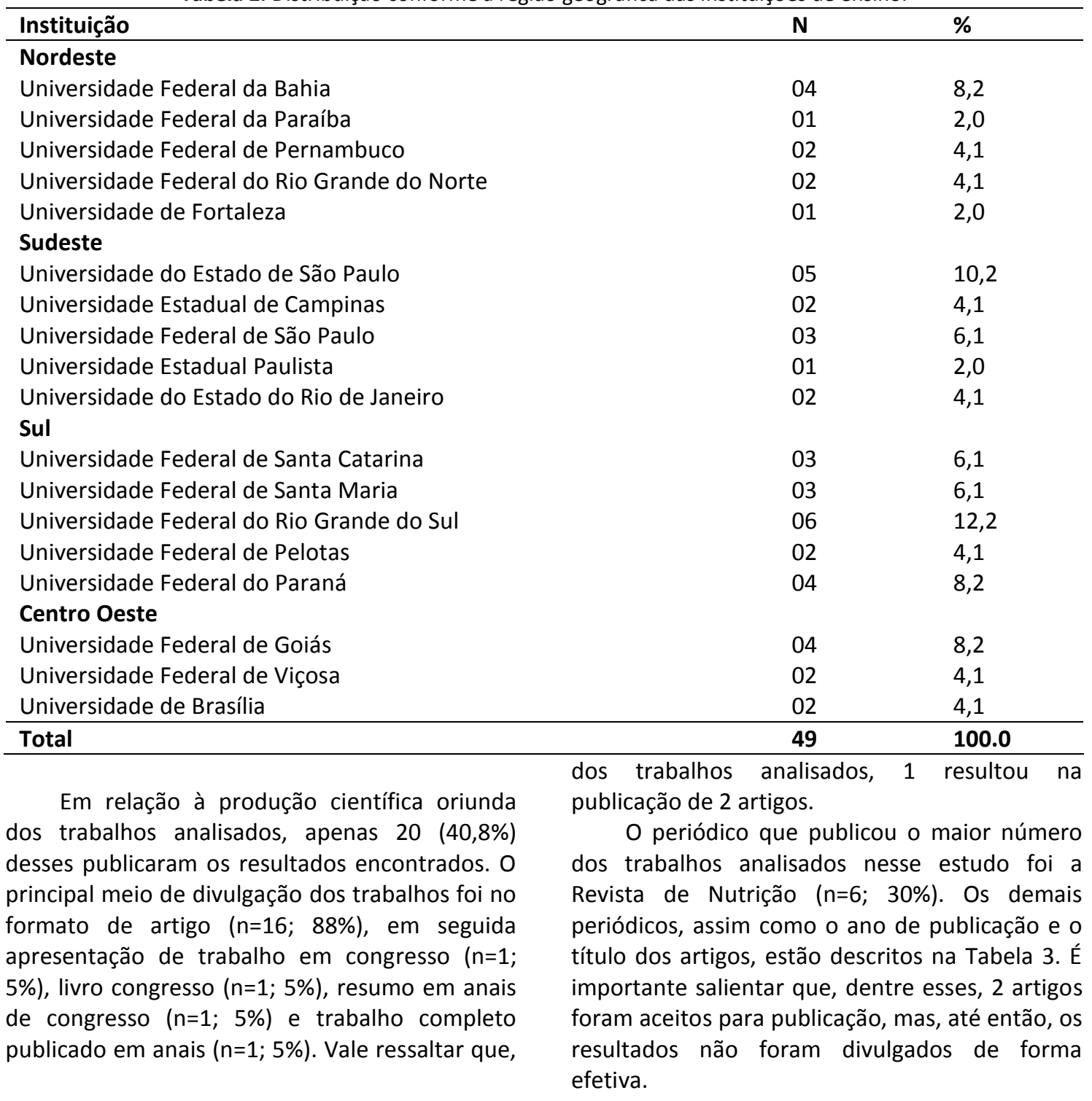

Tabela 3. Artigos publicados e aceitos para publicação, segundo periódico, título e ano.

\begin{tabular}{lll}
\hline Periódico & Título & Ano \\
\hline Annals of Human Biology & Prediction equations for fat and fat-free body mass in 2012 \\
& adolescents, based on body circumferences & \\
Nutrire & Formação do nutricionista atuante no Programa 2012 \\
& Nacional de Alimentação Escolar \\
Revista de Nutrição & Knowledge of educators and dieticians on food and 2013 \\
& nutrition education in the school environment
\end{tabular}

Revista de Nutrição

District of Brazil 
...continuação

Revista Segurança Alimentar e Avaliação do Programa Nacional de Alimentação

Nutricional

Revista de Saúde Pública

Jornal de Pediatria

Revista de Nutrição

Intellèctus

Linhas

BMC Public Health

Revista de Nutrição

Revista de Nutrição

Revista de APS

Cadernos Saúde Coletiva

Revista de Nutrição

Saúde e Sociedade
Escolar: desenvolvimento de um protocolo de indicadores

Redução do uso de açúcar em escolas públicas: ensaio randomizado por conglomerados

Effect of intervention programs in schools to reduce screen time: a meta analysis

Organic food-related educational actions developed

by dieticians in Brazilian municipal schools

Francisco Pompêo do Amaral: médico, jornalista,

2015

professor e escritor científico, político e social no campo da alimentação e nutrição no Brasil

Francisco Pompêo do Amaral: sujeito social e seus

objetos de ensino em prol da alimentação e nutrição no Brasil

Design, randomization and methodology of the

TriAtiva Program to reduce obesity in school children in Southern Brazil

Qualidade da dieta de escolares de 7 a 10 anos do município de São Paulo: associação com o número e os locais de refeições

School menus in Santa Catarina: Evaluation with

respect to the National School Food Program regulations

O Programa Saúde na Escola e as ações de

alimentação e nutrição: uma análise exploratória

Promoção da Alimentação Saudável na Escola: 2015 realidade ou utopia?

Factors associated with overweight among elementary

schoolchildren in Campinas, Sao Paulo, Brazil

Vivências de plantar e comer: a horta escolar como

prática educativa, sob a perspectiva dos educadores
No presente estudo, observou-se que o tema educação nutricional, alimentação e escolares vem sendo trabalho nos programas brasileiros de pós-graduação, por meio de produção de dissertações e teses, sendo o ano de 2014 o mais produtivo. Um dos motivos que pode contribuir para o aumento do interesse na discussão sobre o tema é o elevado número de escolares no sobrepeso e obesidade. Somando-se a isso, os fatores biopsicológicos, socioeconômicos e comportamentais tendem a influenciar essa problemática $^{15}$.

Outro marco fundamental para a ascensão da discussão acerca da alimentação 
escolar foi a inclusão normativa do nutricionista como Responsável Técnico do PNAE. Essa conquista só foi possível em virtude da atuação expressiva dos Conselhos de Alimentação Escolar. Além disso, o aumento no repasse de recursos financeiros através do PNAE reforça a importância da alimentação escolar para a qualidade alimentar e nutricional dos escolares ${ }^{16}$.

Nesse contexto, os resultados desse estudo mostram que o profissional nutricionista está mais inserido no desenvolvimento de trabalhos acerca da temática em questão. Esse achado reforça a inclusão normativa do nutricionista como Responsável Técnico do PNAE, muito embora tenha passado por alguns percalços até conseguir essa autonomia e responsabilidade.

Em 1994, o nutricionista foi apontado como responsável pela elaboração dos cardápios do programa de alimentação escolar, consoante a Lei $n^{\circ} 8.913^{17}$. Somente em 2006 esse profissional foi inserido como responsável técnico do PNAE, de acordo a Resolução no 32, do Fundo Nacional de Desenvolvimento da Educação $(F N D E)^{18}$. Porém, essas atribuições legais foram revogadas e, atualmente, os princípios e diretrizes em vigor estão dispostos na Resolução $n^{\circ} 38^{10}$.

No que se refere às instituições de ensino e, consequentemente, à região geográfica que as mesmas estão inseridas, observa-se nesse estudo que a região Sul $(n=18 ; 36,7 \%)$ se destaca em relação às demais. Por outro lado, pode-se notar que, na Região Norte, não foi encontrado nenhum trabalho acerca do tema em questão.

Corroborando com o achado acima, Chaves e colaboradores apontam que a região Sul possui o maior valor percentual (85\%) de municípios com nutricionistas cadastrados no PNAE/FNDE. Em contrapartida, a região Norte possui o menor percentual de municípios cobertos por nutricionistas cadastrados $(59 \%)^{19}$.

Desse modo, ao passo que esses profissionais estão trabalhando e estudando sobre educação nutricional, alimentação e escolares em suas dissertações e teses, torna-se essencial a divulgação dos resultados encontrados em seus trabalhos, visto que os mesmos devam servir de alguma forma para a sociedade. No entanto, no presente estudo restou evidenciado que, dos trabalhos analisados, apenas 20 (40,8\%) tiveram seus resultados publicados.

É cediço que a publicação dos resultados é o principal veículo de informação e divulgação da pesquisa. A produção científica deve ser útil e contribuir para a população em estudo e para a sociedade como um todo. Além disso, a divulgação dos trabalhos no formato de artigo é um meio rápido e econômico que os pesquisadores possuem para disseminar os resultados encontrados ${ }^{20}$.

A Revista de Nutrição da Pontifícia Universidade Católica de Campinas (Puccamp) foi o principal periódico que publicou os resultados encontrados nas dissertações e teses analisadas nesse estudo ( $n=6 ; 30 \%$ ). Essa revista tem como missão a publicação de trabalhos que contribuam para o estudo da Nutrição em suas diversas subáreas e interfaces.

Uma das formas de avaliar os programas de pós-graduação stricto sensu é por meio do quantitativo e qualidade das produções científicas $^{21}$. A Coordenação de Aperfeiçoamento de Pessoal de Nível Superior (CAPES), no Brasil, avalia e classifica os periódicos científicos, sendo a Revista de Nutrição da Puccamp classificada com o QUALIS B2 na área de Nutrição ${ }^{22}$.

\section{Conclusões}

A temática educação nutricional, alimentação e escolares é muito pertinente para os dias atuais, visto que a alimentação interfere substancialmente no processo saúde-doença do indivíduo. O levantamento bibliométrico do tema permite identificar tendências, padrões de pesquisa, pontos positivos e prováveis carências a serem trabalhadas.

Desse modo, nota-se que os pesquisadores e os programas de pós-graduação têm desenvolvido trabalhos sobre a educação nutricional, alimentação e escolares, os quais buscam contribuir para produção científica e para sociedade.

Assim, para que os trabalhos desenvolvidos nas dissertações e teses surtam impacto na população, é necessária a divulgação dos resultados, sendo esses em qualquer formato, ou, principalmente, em artigo, pelo baixo custo e o fácil acesso da informação. É dever do pesquisador contribuir com o seu conhecimento para a sociedade, sobretudo realizar o feedback com a população em estudo.

\section{Referências}

1. Ministério da Saúde (BR). Secretária de Atenção à Saúde. Departamento de Atenção Básica. Guia Alimentar para População Brasileira. 2a Ed. Brasília (DF): Ministério da Saúde; 2014.

2. Hendrie G, Sohonpal G, Lange K, Golley R. Change in the family food environment is associated with positive dietary change in 
children. Int J Behav Nutr Phys Act. 2013; 10 (4): 1-11.

3. Buck $\mathrm{C}$ et al. Clustering of unhealthy food around German schools and its influence on dietary behavior in school children: a pilot study. International Journal of Behavioral Nutrition and Physical Activity. 2013; 10 (65): 1-10.

4. Instituto Brasileiro de Geografia e Estatística. Pesquisas de Orçamentos Familiares 2008-2009: antropometria e estado nutricional de crianças e adolescentes e adultos no Brasil. Rio de Janeiro: Instituto Brasileiro de Geografia e Estatística; 2010.

4. Silveira JAC, Colugnati FAB, Cocetti $M$, Taddei JAAC. Tendência secular e fatores associados ao excesso de peso entre préescolares brasileiros: PNSN-1989, PNDS-1996 e 2006/07. J. Pediatr. (Rio J.) [Internet]. 2014 Junho; 90 ( 3 ): 258-266.

5. Dutra GF, Kaufmann CC, Pretto ADB, Albernaz EP. Sedentary lifestyle and poor eating habits in childhood:a cohort study. Ciênc. saúde coletiva [Internet]. 2016 Abril; 21( 4 ): 10511059.

6. Wate JT, Snowdon W, Millar L, Nichols M, Mavoa H, Goundar R et al. Adolescent dietary patterns in Fiji and their relationships with standardized body mass index. Int J Behav Nutr Phys Act., 2013; 10(45).

7. Pamela AW et al. Nutrition-Education Program Improves Preschoolers' At-Home Diet: A Group Randomized Trial. Journal of the Academy of Nutrition and Dietetics., 2014; 114(7):10011008.

8. Danelon MAS, Danelon MS, Silva MV da. Serviços de alimentação destinados ao público escolar: análise da convivência do Programa de Alimentação Escolar e das cantinas. Segurança Alimentar e Nutricional, Campinas., 2006; 13(1): 85-94.

9. Ministério da Educação (BR). Resolução CD/FNDE $n^{\circ} 38$ de 16 de julho de 2009. Dispõe sobre o atendimento da alimentação escolar aos alunos da educação básica no Programa Nacional de Alimentação Escolar - PNAE. Diário Oficial da União. Brasília (DF): Ministério da Educação; 2009.

10. Ministérios da Educação e da Saúde (BR). Decreto no. 6.286, de 5 de dezembro de 2007. Institui o Programa Saúde na Escola - PSE, e dá outras providências. Diário Oficial da União. Brasília (DF): Ministérios da Educação e da Saúde; 2007.

11. Freitas MS, Minayo MCS, Ramos LB, Fontes GV, Santos LA, Souza EC et al. Escola: lugar de estudar e de comer. Ciênc. saúde coletiva [Internet]. 2013 Abril; 18( 4 ): 979-985.
12. Salvador GP. Una nueva perspectiva teórica de la bibliometría basada en su dimensión histórica y sus referentes temporales [editorial]. Investig Bibliotecol Arch Bibliotecol Inform. 2016; 30 (70):11-6.

13. Biblioteca Digital Brasileira de Teses e Dissertações (BDTD). Sobre a BDTD. Disponível em: <http://bdtd.ibict.br/vufind/>.

14. Guimarães ACA, Feijó I, Soares A, Fernandes S, Machado Z, Parcias SR. Excesso de peso e obesidade em escolares: associação com fatores biopsicológicos, socioeconômicos e comportamentais. Arq Bras Endocrinol Metab. 2012; 56 (2): 142-148.

15. Peixinho AML. A trajetória do Programa Nacional de Alimentação Escolar no período de 2003-2010: relato do gestor nacional. Ciência \& Saúde Coletiva. 2013; 18 (4): 909-916.

16. Presidência da República (BR). Lei no 8.913 de 12 de julho de 1994 . Dispõe sobre a municipalização da merenda escolar. Diário Oficial da União: Brasília; 1994.

17. Ministério da Educação (BR). Resolução/FNDE/CD/n ${ }^{\circ} 32$ de 10 de agosto de 2006. Estabelece as normas para a execução do Programa Nacional de Alimentação Escolar. Diário Oficial da União: Brasília; 2006.

18. Chaves LG, Santana TCM, Gabriel CG, Vasconcelos FAG de. Reflexões sobre a atuação do nutricionista no Programa Nacional de Alimentação Escolar no Brasil. Ciênc. saúde coletiva [Internet]. 2013; 18( 4 ): 917-926.

19. Brofman PR. A importância das publicações científica. Cogitare Enferm. 2012; 17(3): 419-21.

20. Erdmann AL, Marziale MHP, Pedreira MLG, Lana FCFF, Pagliuca MLF, Padilha MI et al. A avaliação de periódicos científicos qualis e a produção brasileira de artigos da área de enfermagem. Rev Latino-Am Enferm. 2009 Junho 17(3): 403-9.

21. Coordenação de Aperfeiçoamento de Pessoal de Nível Superior (CAPES). Qualis 2014. Disponível em: $<$ https://sucupira.capes.gov.br/sucupira/public/c onsultas/coleta/veiculoPublicacaoQualis/listaCon sultaGeralPeriodicos.jsf> 


\section{Endereço para Correspondência}

Universidade Estadual do Sudoeste da Bahia -

UESB

Av. José Moreira Sobrinho - Jequiezinho, Jequié - BA

CEP.: $45205-490$

e-mail: jamille.marinho@hotmail.com

Recebido em 06/09/2017

Aprovado em 01/11/2017

Publicado em 